\title{
Eary Growth, Pigmentation, Protein Content, and Phenylalanine Ammonia-lyase Activity of Red Curled Lettuces Grown under Different Lighting Conditions
}

\author{
Jeong Wook Heo ${ }^{1}$, Dong Hyeon Kang ${ }^{2}$, Hea Son Bang ${ }^{1}$, Seung Gil Hong ${ }^{1}$, \\ Changhoo Chun ${ }^{3,4 *}$, and Kee Kyung Kang ${ }^{1}$ \\ ${ }^{I}$ Climate Change \& Agroecology Division, Department of Agricultural Environment, National Academy of Agricultural Science, \\ Rural Development Administration, Suwon 441-707, Korea \\ ${ }^{2}$ Farming Automation Division, Department of Agricultural Engineering, National Academy of Agricultural Science, \\ Rural Development Administration, Suwon 441-707, Korea \\ ${ }^{3}$ Departemnt of Plant Science, Seoul National University, Seoul 151-921, Korea \\ ${ }^{4}$ Research Institute for Agriculture and Life Sciences, Seoul National University, Seoul 151-921, Korea
}

\begin{abstract}
Early growth, pigmentation, protein content, and phenylalanine ammonia-lyase (PAL) activity of red curly lettuces (Lactuca sativa L.) grown under different lighting conditions were investigated. Fluorescent lamps (control), blue, red, and blue plus red light-emitting diodes (LEDs) were used as light sources for 10 days. An equal proportion (1:1) of blue and red LEDs was used in the mixed radiation condition. Compared with the control, monochromic red or blue lighting increased fresh and dry weights of 'Ttuksum' and 'Jaju' lettuces. Anthocyanin synthesis was also significantly promoted by the mixed radiation of blue and red LEDs. The mixed radiation also increased the protein content and PAL enzyme activity of 'Ttuksum' leaves by about $200 \%$ compared to other treatments. Anthocyanin content was the highest in lettuces subjected to the mixture radiation of blue and red light treatment, while anthocyanin synthesis was inhibited by monochromic red light. The results of the present study indicate that growth and pigment synthesis in lettuces are significantly enhanced by exposure to mixed radiation from blue and red LEDs.
\end{abstract}

Additional key words: anthocyanin, blue light, light-emitting diode, mixed radiation, red light

\section{Introduction}

Lettuces are available in various cultivars with different leaf colors including light green, dark green, red and spotted. Red curled lettuces have light green leaves with red edges, and their red color is due to the anthocyanin pigment which is a secondary metabolic product. The anthocyanins of red lettuces have anti-oxidant properties. Anthocyanins are members of a class of water-soluble pigments that can be classified as flavonoid and biophenolic compounds. These, together with carotenoids and chlorophylls, are responsible for color in many plants. Effects of light quality on photochemical characteristics such as anthocyanin synthesis and antioxidant properties, have been reported for several different fruits and leafy vegetables (Goins et al., 1997; Kang and Saltveit, 2002; Llorach et al., 2008).

Light-emitting diodes (LEDs) have been considered as a novel light sources for growth and photo-morphogenesis in horticultural plant species because of their small masses, volumes and durability (Brown et al., 1995). These attractive characteristics may make LEDs suitable for use in plant production not only in growth chambers but also in greenhouses. In commercial plant production, light intensity and quality play roles in the establishment of photosynthesis and morphogenesis of many plant species and are essential factors for plant growth and development under greenhouse conditions (Amudha et al., 2005; Heo et al., 2002, 2003, 2006, 2009; Kim et al., 2004). In several plant species, it has been

\footnotetext{
*Corresponding author: changhoo@snu.ac.kr

※ Received 4 October 2011; Revised 4 November 2011; Accepted 8 November 2011. This study was carried out with the support of 'Research Program for Agricultural Science \& Technology Development (Project No. PJ0076662011)', National Academy of Agricultural Science, Rural Development Administration, Republic of Korea. We would like to thank Dr. K.S. Shin of the National Institute of Agricultural Biotechnology, Rural Development Administration, Korea, for his cooperation with the anthocyanin analysis.
} 
suggested that using LEDs as light sources under in vitro or ex vitro conditions may have valuable effects on growth, vegetable quality, or biochemical responses such as increasing of phenolic compound, and ascorbic acid content (Hunter and Burritt, 2004; Ohashi-Kaneko et al., 2007; Qian and Kubota, 2009; Wu et al., 2007).

At the present time, several researchers have reported observing beneficial effects on secondary metabolism in vegetables such as the production of polyphenols or antioxidant properties under controlled environments (Dupont et al., 2000; Llorach et al., 2008). Specifically, regulating the growth and biochemistry of plants by manipulating spectral light qualities using LEDs may increase the amounts of beneficial compounds in red leaf lettuces, which are a relatively rich source of anthocyanin. Therefore, the objective of the present study was to understand physiological characteristics in pigmentation, phenylalanine ammonia-lyase (PAL) enzyme activity, and growth of two red curly lettuce cultivars during early growth stage after transplanting, 'Ttuksum' which is characterized as a with a green leaf before true leaf development, and 'Jaju' which is red leaf lettuce throughout the whole growth stage, under growth chamber conditions with different spectral quality treatments provided by LEDs of blue, red, and blue plus red.

\section{Materials and Methods}

\section{Plant Materials and Cultivation}

Red curly lettuce cultivars (Lactuca sativa L.), 'Ttuksum' and 'Jaju' were germinated in a mixed soil (BM1, Berger Horticulture Co. Ltd., Quebec, Canada) consisting of Canadian sphagnum peat moss, perlite and vermiculite in a greenhouse. The lettuces were sub-irrigated with tap water without any nutritional solutions 3 times during the experimental period throughout the growing period. Seedlings containing two true leaves were used as plant materials and transplanted to 0.3 L plastic pots $(7.5 \mathrm{~cm} \times 6.5 \mathrm{~cm} \times 7.0 \mathrm{~cm})$. The seedlings were placed in a growth chamber under conditions of a 16 $\mathrm{h}$ photoperiod followed by an $8 \mathrm{~h}$ dark period at $25 \pm 1{ }^{\circ} \mathrm{C}$ air temperature and $70 \pm 10 \%$ relative humidity for 10 days.

\section{Light-quality Treatment}

A LED system (GF-320S, Good Feeling Co., Ltd., Sungnam, Korea) with blue, red, and blue plus red lights with 1:1 ratio spectral energy was used for monochromic and mixed lighting. The LED system for light treatments consists of LED sticks with bulbs, a panel $(55 \mathrm{~cm} \times 31 \mathrm{~cm})$, and a main controller $(33.5 \mathrm{~cm} \times 27.5 \mathrm{~cm} \times 10 \mathrm{~cm})$. The LED sticks are mounted on a polycarbonate board $(28 \mathrm{~cm} \times 1$ $\mathrm{cm}$ ) in an array of 20 lamps supplied by a DC power supply. Photosynthetic photon flux (PPF) from the LED system was controlled by adjusting the current using the DC power supply. Minimum energy fluorescent lamps were used as a control. The spectral energy ratio in the mixed radiation treatment of blue and red LEDs was maintained at 1:1. Spectral distributions in relative energy of the improved blue (peak at $450 \mathrm{~nm}$; GaAsAIP, Nichia, Japan) and red (peak at $660 \mathrm{~nm}$; GaAsAl, Sharp, Japan) regions, as determined with a spectroradiometer (Li-1800, LI-COR, Lincoln, NB, USA) in the range of $300-1,100 \mathrm{~nm}$ (Fig. 1). Distance from the light sources to the culture shelf was $50 \mathrm{~cm}$, and $\mathrm{PPF}$ on the empty shelf was kept at $90 \pm 10 \mu \mathrm{mol} \cdot \mathrm{m}^{-2} \cdot \mathrm{s}^{-1}$ in all the light treatments.

\section{PAL Enzyme Activity and Protein Assay}

For measurement of PAL enzyme activity, lettuce leaves of ten plants were homogenized for $2 \mathrm{~min}$ in $15 \mathrm{~mL} 0.1$ $\mathrm{M}$ borate ( $\mathrm{pH}$ 8.0) containing $6 \mathrm{~g}$ polyvinylpyrrolidone, 5 $\mathrm{mM}$ ß-mercaptoethanol and $2 \mathrm{mM}$ EDTA (Jiang and Fu, 1999). The homogenate was centrifuged for $20 \mathrm{~min}$ at 19,000 $\times \mathrm{g}$ and the supernatant was collected for the enzyme assay. PAL enzyme activity was determined by incubating $0.5 \mathrm{~mL}$ of the supernatant in $2 \mathrm{~mL}$ of $0.1 \mathrm{M}$ borate buffer $(\mathrm{pH} 8.0)$ containing $3 \mathrm{mM}$ L-phenylanine for $1 \mathrm{~h}$ at $30^{\circ} \mathrm{C}$. Increase in OD290 nm due to the formation of trans-cinnamate was measured with a spectrophotometer (7310, Shimadzu, Japan). PAL enzyme activity was expressed as the change in OD290 $\mathrm{nm} \cdot \mathrm{h}^{-1} \cdot \mathrm{mg}^{-1}$ protein. Protein was assayed, with bovine serum albumin as the standard, and the assay reagent was prepared by diluting 1 volume of the dye stock with 4 volumes of distilled $\mathrm{H}_{2} \mathrm{O}$ according to the dye-binding method of Bradford (Bradford, 1976).

\section{Pigment Analysis}

Unfolded fresh leaves were randomly collected for anthocyanin determination from the red curled lettuces grown under the specified treatments. The leaves were extracted with 1\% (v/v) cooled methanol (Zhang and Quantick, 1997). The homogenate was centrifuged at $19,000 \times \mathrm{g}$ for $15 \mathrm{~min}$, and diluted with 1\% HCl-methanol to $500 \mathrm{~mL}$. Absorption of the diluents was measured at $530 \mathrm{~nm}$ and anthocyanin contents were expressed as OD530 $\mathrm{nm} \cdot 100 \mathrm{~g}^{-1}$ fresh weight. Chlorophylls and carotenoids were extracted in darkness using the methanol-chloroform $(1: 3 \mathrm{v} / \mathrm{v})$ procedure described by Quail et al. (1976). Chlorophyll a and b, as well as total carotenoid in the chloroform layer, were calculated according to the equation proposed by Wellburn (1994), and the spectrophotometer readings were made at 646 and $663 \mathrm{~nm}$. 
Table 1. Fresh and dry weights of lettuce shoots grown under different light qualities for 10 days.

\begin{tabular}{|c|c|c|c|c|}
\hline \multirow{2}{*}{ Treatments } & \multicolumn{2}{|c|}{ 'Ttuksum' } & \multicolumn{2}{|c|}{ ‘Jaju’ } \\
\hline & Fresh weight (g) & Dry weight (mg) & Fresh weight (g) & Dry weight (mg) \\
\hline Fluorescent lamp (FL) & $2.8 \mathrm{~b}^{\mathrm{z}}$ & $95.3 \mathrm{c}$ & $2.1 \mathrm{ab}$ & $95.0 \mathrm{bc}$ \\
\hline Blue (B) & $2.7 \mathrm{~b}$ & $98.0 \mathrm{c}$ & $2.4 \mathrm{a}$ & $120.5 \mathrm{a}$ \\
\hline $\operatorname{Red}(\mathrm{R})$ & $4.3 \mathrm{a}$ & $179.5 \mathrm{a}$ & $1.8 \mathrm{~b}$ & $90.0 \mathrm{c}$ \\
\hline $\mathrm{BR}(\mathrm{BR})^{\mathrm{y}}$ & $3.2 \mathrm{~b}$ & $142.8 \mathrm{~b}$ & $1.6 \mathrm{~b}$ & $119.3 \mathrm{ab}$ \\
\hline
\end{tabular}

${ }^{z}$ In a column, the mean values followed by a different letter are significantly different at the $5 \%$ level by Duncan's multiple range test.

$\mathrm{y}_{1}: 1$ in spectral energy.

\section{Measurement of Growth and Statistical Analysis}

The lettuces were harvested on day 10 after transplanting for destructive measurement taken on fresh and dry weights. Experiments with two replications per treatment included 20 plants of each cultivar for each of the treatments. The shoot-dry weight of two lettuce cultivars was determined after drying for $48 \mathrm{~h}$ at $70^{\circ} \mathrm{C}$. Duncan's multiple range tests were used to assess the significance of the differences between the treatments using the SAS system (Version 6.21, SAS Institute Inc. Cary, NC, USA). $P$-values $<0.05$ were considered significant.

\section{Results}

\section{Growth}

In 'Ttuksum' lettuce, monochromic red light (R) treatment led to a significant increase of fresh and dry weights 10 days after development of true leaves (Table 1). The dry weight under $\mathrm{R}$ treatment was nearly twice higher than that observed under fluorescent (FL) or blue (B) lighting. The B treatment led to decrease in dry weight of 'Ttuksum', however, the fresh and dry weights of 'Jaju' leaves significantly increased with B treatment as well as with the blue and red lights (BR treatment). In 'Jaju' leaves there was no significant difference in fresh and dry weights between FL and B treatments or between B and BR treatment. The effects of different light treatments on fresh and dry weights in 'Jaju', which continuously produce red pigment in the leaves during the whole growth stage, were less significant than those in 'Ttuksum'.

\section{Pigment Development}

BR treatment increased the amounts of chlorophyll a and $\mathrm{b}$ in the leaves of 'Ttuksum', while blue light alone inhibited chlorophyll synthesis in 'Jaju' leaves (Fig. 1 and Table 2). Higher amounts of carotenoid were found in 'Ttuksum' leaves grown under BR treatment, while carotenoid synthesis in 'Jaju' was not significantly affected by the light qualities

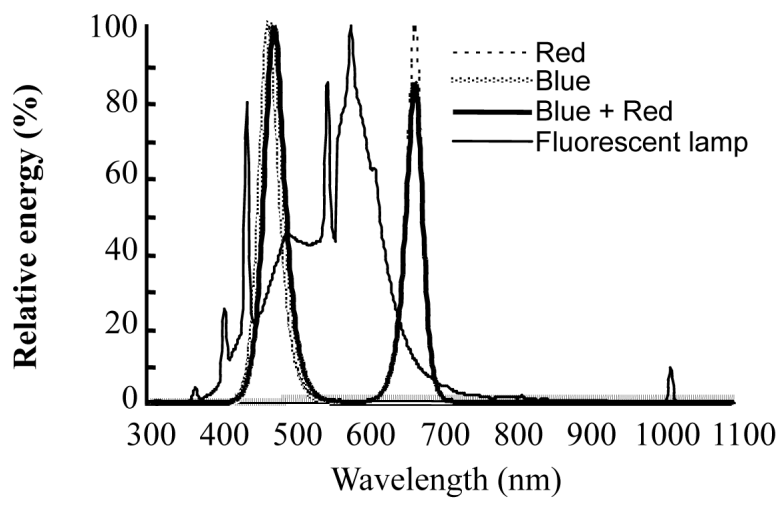

Fig. 1. Spectral distribution in relative energy of blue, red and blue plus red LEDs comparing with conventional fluorescent lamps

between blue and red. In 'Ttuksum' leaves, the R treatment decreased the total chlorophyll/carotenoid ratio, while the B treatment decreased the same ratio in 'Jaju'. However, the different light qualities had no effect on the chlorophyll $\mathrm{a} / \mathrm{b}$ ratio in either of the lettuces.

Upon examining the pigment accumulation after true leaf unfolding in 'Ttuksum', we found that the anthocyanin content was twice higher under BR treatment compared to the control (Fig. 2). BR radiation also stimulated the anthocyanin synthesis in 'Jaju' but blue light significantly inhibited pigment production. Blue light inhibited anthocyanin synthesis in 'Jaju' compared with other light qualities, although fresh and dry weights were significantly promoted by blue light. 'Jaju' lettuces producing red pigment during the whole growth stage were considerably more sensitive to the light stress induced pigmentation when compared to 'Ttuksum' leaves after true leaf development.

\section{PAL Enzyme Activity and Protein Content}

As shown in the anthocyanin accumulation results, PAL enzyme activity and protein content of green curled 'Ttuksum' leaves were also remarkably promoted by the mixed radiation of blue and red lights (Fig. 2). PAL enzyme activity of 'Ttuksum' was more than doubled in the BR mixed treatment 
Table 2. Chlorophyll (Chl.) and carotenoid production $\left(\mathrm{mg} \cdot \mathrm{g}^{-1} \mathrm{FW}\right)$ in the lettuces grown under different light qualities for 10 days.

\begin{tabular}{|c|c|c|c|c|c|}
\hline \multirow{2}{*}{ Treatments } & \multicolumn{5}{|c|}{ 'Ttuksum' } \\
\hline & Chl. a & Chl. b & Chl. $a / b$ & Carotenoid & Chl./Carotenoid \\
\hline Fluorescent lamp (FL) & $3.6 b^{z}$ & $1.1 \mathrm{~b}$ & $3.3 \mathrm{NS}^{\mathrm{y}}$ & $1.4 \mathrm{~b}$ & $3.5 \mathrm{a}$ \\
\hline Blue (B) & $3.8 \mathrm{~b}$ & $1.0 \mathrm{~b}$ & 3.8 & $1.5 \mathrm{~b}$ & $3.2 \mathrm{~b}$ \\
\hline $\operatorname{Red}(\mathrm{R})$ & $3.4 \mathrm{~b}$ & $0.9 \mathrm{~b}$ & 3.8 & $1.5 \mathrm{~b}$ & $2.9 \mathrm{c}$ \\
\hline $\mathrm{BR}(\mathrm{BR})^{\mathrm{x}}$ & $6.9 \mathrm{a}$ & $1.9 \mathrm{a}$ & 3.7 & $2.7 \mathrm{a}$ & $3.2 \mathrm{~b}$ \\
\hline \multirow{2}{*}{ Treatments } & \multicolumn{5}{|c|}{ ‘Jaju’ } \\
\hline & Chl. a & Chl. b & Chl. a/b & Carotenoid & Chl./Carotenoid \\
\hline Fluorescent lamp (FL) & $6.3 a b$ & $1.6 \mathrm{a}$ & $3.9 \mathrm{NS}$ & $2.6 \mathrm{ab}$ & $3.0 \mathrm{a}$ \\
\hline Blue (B) & $4.8 \mathrm{~b}$ & $1.2 \mathrm{~b}$ & 4.1 & $2.1 \mathrm{~b}$ & $2.8 \mathrm{~b}$ \\
\hline $\operatorname{Red}(\mathrm{R})$ & $7.0 \mathrm{a}$ & $1.7 \mathrm{a}$ & 4.1 & $2.9 \mathrm{a}$ & $3.1 \mathrm{a}$ \\
\hline $\mathrm{BR}(\mathrm{BR})$ & $6.2 a b$ & $1.6 \mathrm{a}$ & 4.0 & $2.6 \mathrm{ab}$ & $3.0 \mathrm{a}$ \\
\hline
\end{tabular}

${ }^{z}$ In a column, the mean values followed by a different letter are significantly different at the $5 \%$ level by Duncan's multiple range test.

${ }^{\mathrm{y}} \mathrm{NS}$ : No significant.

$\mathrm{x}_{1: 1}$ in spectral energy.
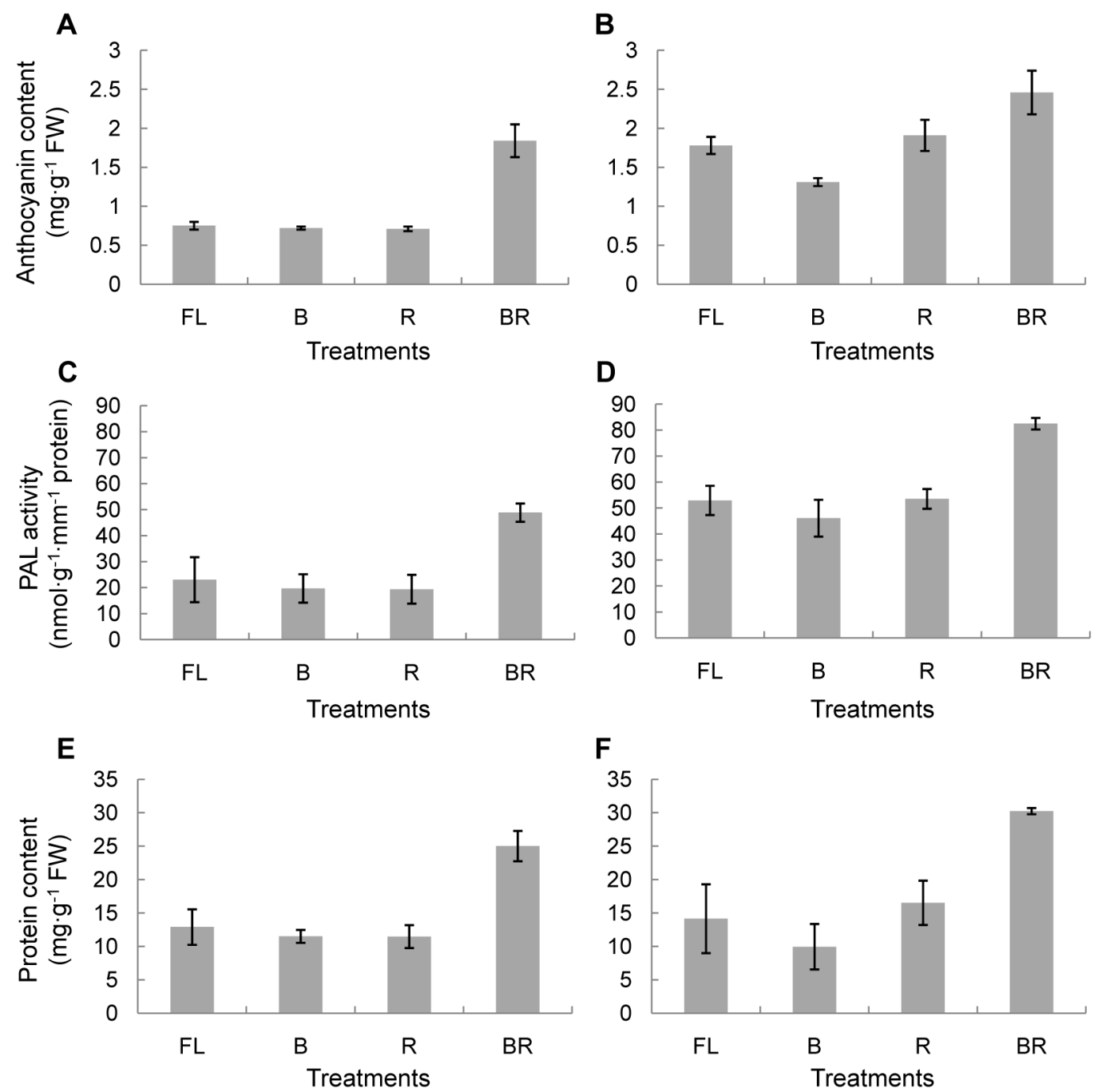

Fig. 2. Anthocyanin content $(A$ and $B), P A L$ activity $(C$ and $D)$ and protein content ( $E$ and $F)$ of the lettuces grown under a $16 \mathrm{~h}$ photoperiod of fluorescent lamp (FL), blue (B), red (R), and blue plus red (BR) and at $90 \pm 10 \mu \mathrm{mol} \cdot \mathrm{m}^{-2} \cdot \mathrm{s}^{-1} \mathrm{PPF}$ for 10 days. Vertical bars represent standard errors. Means values followed by a different letter are significantly different at the $5 \%$ level by Duncan's multiple range test. 
compared with the control. In 'Jaju' leaves, the enzyme activity was also increased in the BR treatment compared with other treatments. Protein content significantly increased in both lettuces grown under the mixture lights as shown in increased anthocyanin content and PAL activity. There was no significantly difference in the light qualities of monochromic blue, red and conventional FL. Protein content was approximately increased by twice in both lettuces when grown under BR as compared to other light treatments.

\section{Discussion}

Increases in dry mass of 'Ttuksum' and 'Jaju' lettuces in early growth stage were induced by exposure to monochromic red or blue light for 10 days after transplanting. The increase in fresh and dry weights in 'Ttuksum' leaves grown under red light was probably due to the expanded leaf area compared to 'Jaju' leaves. However, exposure to the blue light also significantly increased the dry weight in 'Jaju' lettuce as shown in previous research on lettuce growth under blue light (Hoenecke et al., 1992). It was reported that the quantitative growth such as a dry mass increment of in vitro or ex vitro of several plant species was promoted by monochromic red or mixed light of blue and red (Heo et al., 2006, 2010; Hunter and Burritt, 2004; Ohashi-Kaneko et al., 2007). From these results, increase of dry mass in several plants is probably depended on plant species, varieties, specific light quality and/or light intensity responded to various photoreceptor responses such as a phytochrome or cryptochrome.

In contrast, negative effects of red compared to blue light on stem and leaf growth of pea seedlings were described by $\mathrm{Wu}$ et al. (2007). These different growth responses to red or blue light are most likely due to the different actions of phytochrome and cryptochrome photoreceptors, as suggested by previous studies (Doi et al., 2004; Heo et al., 2002; Smith, 2000).

Anthocyanins that accumulate in plants do not participate in primary photosynthetic reactions in chloroplasts, and formation of anthocyanins is a genetically determined by phytochrome mediated process requiring photosynthetic activity (Meng et al., 2004; Strazalkowska et al., 1989). Light is one of the main determinants of anthocyanin production, and anthocyanin biosynthesis is strongly dependent on light intensity or light quality (Kataoka et al., 2003; Merzlyak and Chivkunova, 2000). In particular, when the different ecotypes were kept at different R/FR ratios, the anthocyanin level was significantly increased under higher R/FR ratios, and the pigment levels were almost the same under low R/FR ratios (Alokam et al., 2002). It has also been reported that anthocyanin accumulation in maize seedlings is controlled by Chalcone synthase, Dihydroquercetin reductase, Bronze 1 , and Bronze 2 genes in the biosynthetic pathway during inductive light treatment (Taylor and Briggs, 1990). In 'Ttuksum' and 'Jaju' lettuces, regardless of red coloring before or after true leaf development, anthocyanin accumulation was promoted by BR treatment and inhibited by B treatment. The blue light effect on plant pigmentation was mediated by the cryptochrome, which is a photoreceptor which is a generic name for all putative pigments with high absorption of blue, UV-A and UV-B radiation (Jenkins, 1997). The significant increase in anthocyanin accumulation under BR treatment in both lettuce cultivars as compared with monochromic blue light is probably due to a synergistic interaction of blue and red light receptors controlled by certain genes involved in pigmentation of plant species.

Carotenoid accumulation in 'Ttuksum' lettuce was improved after BR treatment and depressed under blue, red, and fluorescent lamp lighting, while the carotenoid content of 'Jaju' which has red color leaves during the whole growth stage decreased under the blue light mediated cryptochrome group. The amounts of anthocyanin, chlorophyll, and carotenoids were higher in 'Jaju' compared to 'Ttuksum' leaves grown under different light conditions. This means that pigmentation in the lettuces is probably due to the cultivar characteristics and light exposure provided by LEDs. The accumulation of chlorophyll and carotenoid pigments in leafy vegetables is also sensitive to light conditions (Caldwell and Britz, 2006; Qian and Kubota, 2009). The differences in pigmentation of the lettuces are probably affected by the different coloring stages either at the time of true leaf unfolding or during the whole growth stage. Previous studies found that under high-light conditions the content of carotenoids in apples is retained and the synthesis of these pigments is activated (Strazalkowska et al., 1989). Carotenoids are powerful quenchers of excited states and singlet oxygen, and may function as antioxidants (Biswall, 1995). This may help explain the protection of the photosynthetic apparatus under different light conditions.

Anthocyanins are synthesized from hexose through the flavonoid pathway, and PAL has been studied as a rate limiting enzyme of this pathway in strawberry (Given et al., 1988). PAL is the first enzyme in phenylpropanoid metabolism, of which anthocyanin is a product (Dixon and Paiva, 1995). The PAL enzyme activity in 'Ttuksum' lettuce was remarkably increased after the BR treatment. During BR treatment, phenylpropanoid metabolism in 'Ttuksum' is probably more active compared with other light qualities, and large amounts of biophenolic compounds such as anthocyanin and carotenoid are produced. Although other enzymes related to pigmentation 
have not been previously assayed, the present study suggests that BR mixed light has a significant effect on pigment synthesis and growth in red curled lettuces of different coloring stages. Synthesis of light inducible protein in the lettuce leaves is also stimulated by the mixture light quality rather than monochromic blue and red. Pigmentation or protein synthesis in several plant species is probably stimulated by mixture light qualities rather than monochromic qualities (Lopez-Figueroa and Niell, 1990; Adamska et al., 1992).

These results suggest that mixed radiation of blue and red provided by LEDs significantly stimulates anthocyanin production with higher enzyme activity and protein content of the red curled lettuce cultivars on early growth stage compared to monochromic blue light. However, whether physiological characteristics of light-stress induced enzymes related to pigmentation are affected by light quality and whether mixed radiation can promote the pigmentation through the whole growth stage of the lettuces grown under higher air temperatures is unknown, and further studies are required.

\section{Literature Cited}

Adamska, I., I. Ohad, and K. Kloppstech. 1992. Synthesis of the early light-inducible protein is controlled by blue light and related to light stress. Proc. Natl. Acad. Sci. 89:2610-2613.

Alokam, S., C.C. Chinnappa, and D.M. Reid. 2002. Red/far-red light mediated stem elongation and anthocyanin accumulation in Stellaria longipes: Differential response of alpine and prairie ecotypes. Can. J. Bot. 80:72-81.

Amudha, P., M. Jayakumar, and G. Kulandaivelu. 2005. Impacts of ambient solar UV (280-400 $\mathrm{nm}$ ) radiation on three tropical legumes. J. Plant Biol. 48:284-291.

Biswall, B. 1995. Carotenoid catabolism during leaf senescence and its control by light. J. Photochem. Photobiol. Biol. 30:3-14.

Bradford, M.M. 1976. A rapid and sensitive method for the quantitation of microgram quantities of protein utilizing the principle of protein-dye binding. Anal. Biochem. 72:248-254.

Brown, C.S., A.C. Schuerger, and J.C. Sagar. 1995. Growth and photomorphogenesis of pepper plants under red light-emitting diodes with supplemental blue or far-red lighting. J. Amer. Soc. Hort. Sci. 120:808-813.

Caldwell, C.R. and S.J. Britz. 2006. Effect of supplemental ultraviolet radiation on the carotenoid and chlorophyll composition of green house-grown leaf lettuce (Lactuca sativa L.) cultivars. J. Food Composition Analysis 19:637-644.

Dixon, R.A. and N.L. Paiva. 1995. Stress-induced phenylpropanoid metabolism. Plant Cell 7:1085-1097.

Doi, M., A. Shigenaka, T. Emi, T. Kinoshita, and K. Shimazaki. 2004. A transgene encoding a blue-light receptor, phot1, restores blue-light responses in the Arabidopsis phot1 phot2 double mutant. J. Exp. Bot. 55:517-523.

Dupont, S., Z. Mondi, G. Willamson, and K. Price. 2000. Effect of variety, processing, and storage on the flavonoid glycoside and composition of lettuce and chicory. J. Agri. Food Chem.
48:3957-3964.

Given, N.K., M.A. Venis, and D. Grieson. 1988. Phenylalanine ammonia-lyase activity and anthocyanin synthesis in ripening strawberry fruit. J. Plant Physiol. 133:25-30.

Goins, G.D., N.C. Yoroi, M.M. Sanwo, and C.S. Brown. 1997. Photomorphogenesis, photosynthesis, and seed yield of wheat plants grown under red light-emitting diodes (LEDs) with and without supplemental blue lighting. J. Exp. Bot. 312:1407-1413.

Heo, J.W., C.W. Lee, C. Chakrabarty, and K.Y. Paek. 2002. Growth responses of marigold and salvia bedding plants as affected by monochromic or mixture radiation provided by a light-emitting diode (LED). Plant Growth Regul. 38:225-230.

Heo, J.W., C.W. Lee, H.N. Murthy, and K.Y. Paek. 2003. Influence of light quality and photoperiod on flowering of Cyclamen persicum Mill. cv. 'Dixie White'. Plant Growth Regul. 40:7-10.

Heo, J.W., C.W. Lee, and K.Y. Paek. 2006. Influence of mixed LED radiation on the growth of annual plants. J. Plant Biol. 49:286-290.

Heo, J.W., Y.B. Lee, D.B. Lee, and C. Chun. 2009. Light quality affects the growth, net photosynthetic rate, and ethylene production of ageratum, African marigold, and salvia seedlings. Kor. J. Hort. Sci. Technol. 27: 187-193.

Heo, J.W., Y.B. Lee, Y.S. Chang, J.T. Lee, and D.B. Lee. 2010. Effects of light quality and lighting type using an LED chamber system on Chrysanthemum growth and development cultured in vitro. Kor. Soc. Agri. Environ. 29:374-380.

Hoenecke, M.E., R.J. Bula, and T.W. Tibbitts. 1992. Importance of "blue" photon levels for lettuce seedlings grown under red-light-emitting diodes. Hort. Sci. 27:427-430.

Hunter, D.C. and D.J. Burritt. 2004. Light quality influences adventitious shoot production from cotyledon explants of lettuce (Lactuca sativa L.). In Vitro Cell Dev. Biol. Plant 40:215-220

Jenkins, G.I. 1997. UV and blue light signal transduction in Arabidopsis. Plant Cell Environ. 20:773-778.

Jiang, Y.M. and J.R. Fu. 1999. Biochemical and physiological changes involved in browning of litchi fruit caused by water. J. Hort. Sci. Biotechnol. 74:43-46.

Kataoka, I., A. Sugiyama, and K. Beppu. 2003. Role of ultra violet radiation in accumulation of anthocyanin in berries of 'Gros Colman' grapes (Vitis vinifera L.). J. Japan. Soc. Hort. Sci. 72:1-6.

Kang, H.M. and E. Saltveit. 2002. Antioxidant capacity of lettuce leaf tissue increased after wounding. Agri. Food Chem. 50:7536-7541.

Kim, H.H., G.D. Goins, R.M. Wheeler, and J.C. Sager. 2004. Green-light supplementation for enhanced lettuce growth under red- and blue-light-emitting diodes. HortScience 39:1617-1622.

Llorach, R., A. Martinez-Sanchez, A. Francisco, M.I. Gil, and F. Ferreres. 2008. Characterization of polyphenols and antioxidant properties of five lettuce varieties and escarole. Food Chem. 108:1028-1038.

Lopez-Figueroa, F. and F.X. Niell. 1990. Effects of light quality on chlorophyll and biliprotein accumulation in seaweeds. Marine Biol. 104:321-327.

Meng, X.C., T. Xing, and X.J. Wang. 2004. The role of light 
in the regulation of anthocyanin accumulation in gerbera hybrid. Plant Growth Regul. 44:243-250.

Merzlyak, M.N. and O.B. Chivkunova. 2000. Light-stress-induced pigment changes and evidence for anthocyanin photoprotection in apples. J. Photochem. Photobiol. Biol. 55:155-163.

Ohashi-Kaneko, K., M. Takase, N. Kon, K. Fujiwara, and K. Kurata. 2007. Effects of light quality on growth and vegetable quality in leaf lettuce, spinach and komatsuna. Environ. Control Biol. 45:189-198.

Smith, H. 2000. Phytochromes and light signal perception by plants - An emerging synthesis. Nature 407:585-591.

Strazalkowska, C., D. Slawinsks, A. Slawinski, and J. Slawinski. 1989. Photoinduced delayed luminescence from certain plant tissues containing anthocyanins, betacyanins and chlorophyll. J. Photochem. Photobiol. Biol. 3:259-268.

Taylor, L.P. and W.R. Briggs. 1990. Genetic regulation and photocontrol of anthocyanin accumulation in maize seedlings. Plant Cell 2:115-127.
Qian, L. and C. Kubota. 2009. Effects of supplemental light quality on growth and phytochemicals of baby leaf lettuce. Environ. Exp. Bot. 67:59-64.

Quail, P.H., E.A. Gallagher, and A.R. Wellburn. 1976. Membraneassociated phytochrome: Non-coincidence with plastid membrane marker profiles on sucrose gradients. Photochem. Photobiol. 24:495-498.

Wellburn, A.R. 1994. The spectral determination of chlorophylls $a$ and $b$, as well as total carotenoids, using various solvents with spectrophotometers of different resolution. Plant Physiol. 144:307-313.

Wu, M.C., C.Y. Hou, C.M. Jiang, Y.T. Wang, C.Y. Wang, H.H. Chen, and H.M. Chang. 2007. A novel approach of LED light radiation improves the antioxidant activity of pea seedlings. Food Chem. 101:1753-1758.

Zhang, D. and P.C. Quantick. 1997. Effects of chitosan coating on enzymatic browning and decay during postharvest storage of litchi (Litchi chinensis Sonn.). Biol. Technol. 12:195-202. 